\title{
Recent recommendations for prevention of post dural puncture headache in pregnant females undergoing cesarean section \\ E.F.Makram, M.A.Khashaba, and N.S.Mohamed
}

Anesthesiology, intensive care, Dept., Faculty of Medicine, Benha Univ., Benha, Egypt

E-mail: neama.samy48@gmail.com

\begin{abstract}
Context: Post-dural headache puncture (PDPH) is a frequent consequence after lumbar puncture. PDPH usually has frontal/occipital, postural headaches that are worse while they are standing and better when they are resting. Symptoms associated with stiff neck, loss of hearing, tinnitus, photophobia and nausea. The prevalence of PDPH in pregnant women is greater. This research seeks to examine the latest update on the prevention of post-dural headache puncture for pregnant women in the caesarean section. Methods: The search in MEDLINE, Embase, Pubmed and CINAHL Plus was conducted in the same periodical with the following mediacl terms: "post-dural; cramp; headache; caesarean section," encompassing articles 2000-2020, 2012; Articles other than English are excluded from the review. Results and findings: Headache treatment after accidental hard puncture varies. This diversity extends to the literature with various PDPHA definitions and epidural blood patch indications. More importantly, the absence of follow-up of the patients following accidental dural puncture was a survey addressed to members of the Society for Obstetric Anesthesia and Perinatology. Due to contradictory facts and views, a documented procedure is essential and all departmental members have to be followed.
\end{abstract}

Keywords: Post dural, Puncture, Headache, Cesarean section.

\section{Introduction}

PDPH is a major iatrogenic source of patient morbidity in contemporary anaesthesia, pain management following attempted epidural block and spinal taps. The incidence of dural puncture in skilled hands varies from 0.16 to 1.3 per cent. After attempted epidural blocks with big boron needles, post-dural punching headache occurs in 16 percent-86 percent of cases [1].

The incidence of PDPH after ADP in the obstetrical population ranges from $80-86$ percent. In non-obstetric patients there are minimal data on DP [2].

Dural puncture is an invasive technique frequently used for many reasons, such as diagnosis of lumbar puncture, spinal anesthésy, myelography and intrathecal chemotherapy. However, in the practise of anaesthesia, accidental dural puncture may also occur for different reasons, including postoperative and laboratory relief, in addition to purposeful dural puncture, as in spinal anaesthesia. Carrie and Collins define headache post-hard puncture (PDPH) as "Headache after dural puncture and significantly affects post-operative patients, i.e. headache which is not just postural but continues at any intensity level for over 24 hours or at any time so severe that the patient is unable to maintain a right position[3].

August Bier made a historical reference to PDPH in 1899 when he provided a firsthand description of his headache and suffered from a vertebral anaesthesia on the request of his assistant. Dr. Bier characterised the headache as a sensation of extremely high head pressure and mild dizziness when it rapidly rises out of the chair. The most significant indication of PDPH was also stated as follows: "When I lay down horizontally, the symptoms instantly vanished and returned back when I got upright [4].
After lumbar puncture, PDPH is a frequent consequence. PDPH is a common problem. PDPH usually has frontal/occipital, postural headaches that are worse while they are standing and better when they are resting. Symptoms associated with stiff neck, loss of hearing, tinnitus, photophobia and nausea. The prevalence of PDPH in pregnant women is higher[5].

The cause of PDPH is not completely sure. The best explanation is that low CSF pressure caused by CSF leakage via dural or arachnoid rupture; a leakage that surpasses the production rate of CSF. $4 \mathrm{~A}$ decrease in CSF volume of less than 10 percent may produce orthostatic headache. Two main theoretical processes are available to explain PDPH. Firstly, the meningeal arteries are reflex vasodilated because of the decreased CSF pressure. The second is traction of the intracranial structures which are sensitive to pain in the straight posture. Traction of $\mathrm{C} 1, \mathrm{C} 2$ and $\mathrm{C} 3$ upper cervical nerves produces discomfort in the neck and shoulders. Traction of the fifth cranial nerve produces headaches on the front. Visual problems are caused by traction on the sixth cranial nerve. Pain is caused to traction of the ninth and tenth cranial nerves in the occipital area [6].

The third theory concerns the function of substance $\mathrm{P}$ and neurokininin-1 receptor regulation (NK1R). According to the International Headache Society, PDPHA has no other cause than a headache which occurs within 5 days after hard puncture. It typically involves stiffness of the neck and subjective hearing problems. 4 It also typically spontaneously remits after 2 weeks or after an epidural blood patch has been used to plug the leak [7].

This research is aimed at evaluating the latest update on post-duration puncture prevention in pregnant women who have caesarean surgery. 


\section{Methods}

This is a review article, The search was performed in MEDLINE, Embase, Pubmed and CINAHL Plus in the same date range with the following mediacl terms: "post dural; puncture; headache; cesarean section.",including articles from 2000 to 2020, Excluded articles from review are those of langauge other than English.

\section{Results}

Management of PDPH, fig. (1).

\subsection{Management}

Not all patients will require hospital admission, but for those who do, routine ward monitoring may be appropriate assuming the correct diagnosis of PDPH has been made. For those with evidence of neurological compromise, regular neurological observations may be warranted and will be advised by medical staff. If invasive intervention is required (i.e. epidural blood patch), then hospital admission will be required. Management options are outlined below:
Written information to facilitate discussion should be provided.

\subsection{Psychological reassurance}

Patients who develop post-Dural puncture headache may suffer a wide range of bad emotional responses from misery and tears to anger and panic. It is important both from a clinical and medico-legal point of view, to discuss the possibility of headache before a procedure is undertaken that has a risk of this complication. Even this discussion will not prepare the patient for the sensations he or she feels when the headache develops. [8]

Obstetric patients are particularly prone to develop this complication, especially as they expect to feel well and happy and to be able to look after their new baby. It is important to give the mother a thorough explanation of the reason for the headache, the expected time, course, and the therapeutic options available. Regular review is essential to monitor the course and therapeutic maneuvers undertaken. [8]

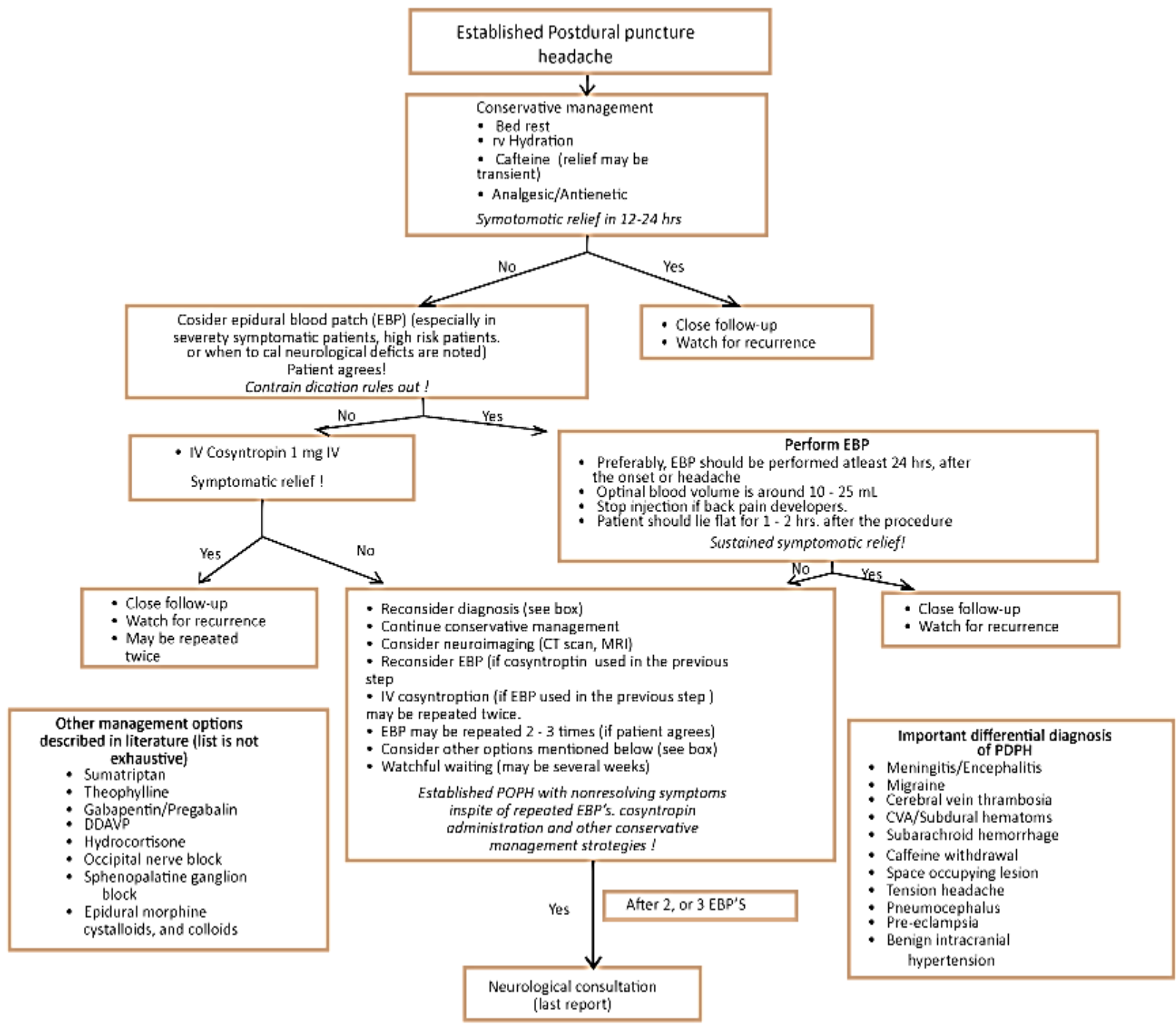

Fig. (1) Algorithm For Managrment Of Post Dural Puncture Headache (Pdph). 


\subsection{Conservative}

Aims to relieve symptoms while waiting for the dural tear to heal by itself It includes:

\subsection{Simple Analgesics}

Simple analgesics such as paracetamol and nonsteroidal anti-inflammatories (NSAIDs), in conjunction with anti-emetics, are the mainstay of PDPH management and have evidence to support their routine use in PDPH. [9]

This Regular paracetamol and NSAIDS (if not contraindicated) may be enough in mild cases. A weak opioid such as codeine, as required, is usually needed as well in moderate and severe cases. Laxatives should be prescribed in conjunction with codeine to prevent constipation. [9]

\subsection{Hydration}

Although there is no evidence to support the therapeutic effect of vigorous hydration, no patient with PDPH should be allowed to become dehydrated and adequate fluid intake should be encouraged.If patients are euvolemic, CSF production is anticipated to be sufficient and increased fluid intake would not be expected to increase its production. [10]

\subsection{Posture}

There is no evidence to support bed rest or specific postures following PDPH. Although it relieves the symptoms, it does not prevent them.

Patients thus should be encouraged to adopt the position which they find most comfortable, including lying flat, as a reduction in analgesic requirements decreases the likelihood of pursuing invasive treatment. It should be kept in mind that post-partum patients are in a hypercoagulable state, so prolonged bed rest may increase risk of venous thromboembolism. Adequate VTE prophylaxis should be prescribed if indicated. So Patients whose headache is severe enough to make them bedridden should be given elasticated stockings and prescribed clexane, around $6 \mathrm{pm}$, in order not to delay a possible EBP the next day [10].

\subsection{Abdominal Binder}

A tight abdominal binder raises the intraabdominal pressure. The elevated intra- abdominal pressure is transmitted to the epidural space and may relieve the headache. Unfortunately, tight binders are uncomfortable and are not used very often in current practice. Few units recommend this approach. [11]

\subsection{Pharmacological Treatment}

Methylxanthine derivatives such as caffeine and aminophylline are the most commonly used drugs for the treatment of PDPH. Caffeine use was introduced in 1949. [11]

\section{Caffeine}

Caffeine is a central nervous system stimulant that poses other properties produces cerebral vasoconstriction. Although previously thought to be of some benefit recent evidence suggests that caffeine intake does not provide a clinically significant improvement and may in fact cause more problems, such as maternal insomnia or neonatal irritability [11].

\section{Adrenocorticotrophic hormone}

Adrenocorticotrophic hormone is another drug, which is being investigated for treating PDPH. It has been administered as an infusion $(1.5 \mu \mathrm{g} \mathrm{kg})$. IT acts by two mechanisms - first, it increases pain threshold by increasing beta-endorphin levels, secondly, it increases CSF production via sodium transporters. Ghai reported $80 \%$ pain relief in a patient who had previously failed conservative therapy after 4 doses of intramuscular ACTH given for 2 days. [9]

\subsection{Cosyntropin}

A recent study using cosyntropin which is an ACTH analog reported remarkable efficacy and need for EBP and significant prolongation of the time from ADP to the occurrence of PDPH but has yet to be independently confirmed. [9]

\subsection{Other drugs}

Both pregabalin and gabapentin have been shown to have a clinical benefit, with pregabalin being the more effective of the two. 5HT agonists (e.g.sumatriptan), DDAVP, theophyline, and hydrocortisone have also been used in the treatment of PDPH, but again there is limited evidence to support their routine use in PDPH treatment. Despite nonconclusive recommendations regarding the efficacy of steroids, one hospital reported their use. [9]

\subsection{Sumatriptan}

Sumatriptan is a 5-HT1D receptor agonist that promotes cerebral vasoconstriction, the same way as caffeine do. Sumatriptan is advocated for the management of migraine and recently, for post-dural puncture headache. There have been only a few case reports where Sumatriptan was used successfully to manage post-dural puncture headache. However, a recent controlled trial found no evidence of benefit from Sumatriptan for the conservative management of post-dural puncture headache. [12]

\subsection{Gabapentin}

Gabapentin is an antiepileptic drug (AED) with analgesic properties. It is approved by the Food and Drug Administration (FDA) for adjunctive treatment of partial epilepsy and management of post herpetic neuralgia. The mechanism by which gabapentin exerts its analgesic action is unknown.

Indications of gabapentin treatment also include: Post herpetic neuralgia, partial seizures, painful diabetic neuropathy, social phobia, anxiety disorders, acquired pendular nystagmus, essential tremor, generalized tonic-clonic seizures, migraine headaches prophylaxis, Parkinsonism, refractory spasticity, restless leg syndrome, phantom limb pain, 
spinal cord injury-related pain, Guillain-Barre-related pain, acute post-mastectomy pain, postmenopausal hot flashes, bipolar mood disorders, panic disorder, Cocaine dependence (Grade D/I),insomnia disorders, posttraumatic stress disorder, irritable bowel syndrome, Trigeminal neuralgia \& other types of neuropathic pain [12].

\subsection{Neostigmine}

Neostigmine methylsulphate is used in the treatment of myasthenia gravis in usual doses of 1 to $2.5 \mathrm{mg}$ daily given in divided doses by subcutaneous, intramuscular, or intravenous injection according to the severity of the condition. Neostigmine is used in conditions of urinary bladder atony due to post anesthetic depression or to neurological disorders. [13]

The possible pathways and mechanisms by which the neostigmine/atropine combination acts to resolve PDPH are shown in Figure20. Following World Health Organization recommendations, breastfeeding was withheld for 24 hours after the last dose of neostigmine/atropine for the safety of the newborn. As no participants required $>2$ doses, breastfeeding was resumed within a relatively short average time of 36 hours after the start of the study intervention. The clinical side effects associated with neostigmine/atropine were primarily cholinergic effects of neostigmine such as abdominal cramps, muscle twitches, and urinary bladder hyperactivity [13].

\subsection{Invasive measures}

Epidural saline: There is a potential danger of an autologous epidural blood patch for the treatment of post- dural puncture headache. The immediate resolution of the headache with a blood patch is due to thecal compression raising the CSF pressure. An epidural injection of saline would also produce the same mass effect and restore normal CSF dynamics. As saline is a relatively inert and sterile solution, epidural saline bolus or infusion appears to be an attractive alternative. Regimens that have been advocated include:

- 1.0-1.5 liter of epidural Hartmanns solution over $24 \mathrm{~h}$, starting on the first day after dural puncture

- Up to 35 ml.h-1 of epidural saline or Hartmanns solution for $24-48 \mathrm{~h}$, or after development of the headache.

- A single $30 \mathrm{ml}$ bolus of epidural saline after development of headache.

- Reduction in the leak would allow the dura to repair. [14]

However, observations of the pressures produced in the subarachnoid and epidural space show that, despite a large rise in epidural pressure, the consequent rise in subarachnoid pressure maintains the differential pressure across the dura. The pressure rise is also not sustained and is dissipated within $10 \mathrm{~min}$. The saline may induce an inflammatory reaction within the epidural space, promoting closure of the dural perforation. Histological studies have not demonstrated an inflammatory response following epidural Dextran 40 administration, however, in contrast to an autologous blood patch. [14]

There are many case reports describing the success of epidural saline, comparative trials with epidural blood patches have not demonstrated the long-term efficacy of epidural saline placement. It is difficult to conclude from the evidence, that epidural saline administration will restore normal CSF dynamics. [14]

Epidural Dextran: Those studies that recommend Dextran 40, either as an infusion or as a bolus, conclude that the high molecular weight and viscosity of Dextran 40 slows its removal from the epidural space. The sustained tamponade around the dural perforation allows spontaneous closure. However, it is unlikely that Dextran 40 will act differently to saline in the epidural space. Any pressure rise within the subarachnoid space would, like saline, be only transient. Histological inspection of the epidural space after administration of Dextran 40, does not demonstrate any inflammatory response that would promote the healing process. The evidence for the administration of epidural Dextran to treat postdural puncture headache is not yet proven, rendering it as an arguable line of treatment [15].

\subsection{Epidurally administered opioids}

A number of authors have advocated the use of epidural, intra thecal or parenteral morphine, however, the majority of these reports are either case reports or inadequately controlled trials. Some of the studies used epidural morphine after the onset of headache; others used epidural or intrathecal morphine as prophylaxis or in combination with an intrathecal catheter. [15].

A reported trial of intrathecal fentanyl as prophylaxis found no evidence of a reduction in the incidence of post-spinal headache after dural puncture with a 25-gauge spinal needle. Also the use of epidural opioids, either as a single injection or continuous infusion, is an important analgesic option for the treatment of postoperative pain \& despite some of the side effects associated with epidural opioid administration, there are many advantages of using epidural opioids for analgesia including some data that suggest an improvement in some clinically oriented patient outcomes. [9]

\section{Discussion}

The patient should stay supine for 2 hours after the operation. Routine ward observations are suitable and women may be released when they are mobilised and examined by an anestheticist. While the most successful treatment was shown for patients with an EBP > 24-48 hours following the dural punch, it should not be delayed before this period if patients are substantially symptomatic. If repeat EDB is necessary, 
fluoroscopic / CT guided operations / imaging may be taken into account. [9]

Epidural blood patches include the identification of an epidural space and autologous blood injections of $15 \mathrm{~mL}$ to $20 \mathrm{~mL}$. Gormley is linked to the use of an epidural blood patch for treating headache after hard puncture, which has shown that patients with bloody lumbar puncture have a reduced chance of getting headache than those who have not. Gormley then examined 7 headache patients using dural puncture, 1 of whom was himself. All experienced headache, which was alleviated with $2 \mathrm{~mL}$ to $3 \mathrm{ml}$ of blood epidural injection. The blood quantity for an epidural blood patch has been investigated. There were many case series with epidural injections of $6 \mathrm{~mL}$ to $50 \mathrm{~mL}$ of blood. [16].

In a randomised trial, 120 participants with an epidural needle and headache accidental durum puncture were assigned to get an epidural blood patch. The blood patch volume was either $15 \mathrm{~mL}, 20 \mathrm{~mL}$ or $30 \mathrm{~mL}$. The frequency of partial alleviation was $51 \%$, $41 \%$ and $41 \%$ respectively, and of total relief was $10 \%, 32 \%$, and $26 \%$, respectively. If a patient complained during injection of acute back discomfort, the ultimate amount of blood utilised was restricted. Thus only $81 \%$ of participants in the $20-\mathrm{mL}$ Group got the entire $20 \mathrm{~mL}$, whereas only $54 \%$ of the participants in the $30-\mathrm{mL}$ group received the full $20 \mathrm{~mL}$. The main aspect of this research is that $20 \mathrm{ml}$ of blood is the optimum amount of an epidural blood patch, provided that throughout the injection the patient has no back discomfort or leg pain. There was no benefit in raising the volume because of back discomfort in higher quantities[17].

Epidural blood patch improves vision disorders with the involvement of the cranial nerve IV. It also enhances the hearing implication of cranial nerve VIII. There is still some residual hearing change, which the patient does not detect or is clinically significant.

A patient is less likely to acquire persistent headache. Approximately $10 \%$ of the epidural blood patches of the SCORE experiment must be redone owing to a recurrence to symptoms.

Complications of epidural blood patches include back discomfort with an estimated $80 \%$ prevalence of back pain.

Another frequent consequence is root pain caused by the inflammatory reaction by blood clots and compression of the nervous roots in the epidural region. Other rare complications: chronic arachnoiditis sticky. Chronic arachnoiditis is an uncommon disease that involves back pain, discomfort in the legs, neurological abnormalities and alterations in RMD. Also reported is spinal subdural hematoma needing immediate neurochirurgical repair. [18]

The issue with epidural blood patches is whether the success of sequential epidural catheters may be impaired. 29 individuals with PDPHA and epidural blood patches were matched to 55 patients with dural puncture and no epidural blood patch in a retrospective analysis. There was no difference between the 2 groups in the success of the following epidural anaesthetics. After an epidural blood patch, a patient had a failed epidural for an unintentional dural puncture. Scarring occurred on the patient's epidurogram, with the contrast material limited to T12 to L2. Alternative blood agents such as fibrinous glue have been suggested to heal spinal piercing. [19].

Cranial dural drilling is often effectively healed with it. The fibrin glue may be positioned blindly or in the event of lumbar perforation with CT-guided percutaneous injection [16].

However, with this surgery, there is a danger of developing aseptic meningitis. Moreover, producers have lately cautioned against using some kinds of tissue glue where nerve tissue may be exposed. After inadvertent dural drilling with a Tuohy needle, the positioning of a spinal catheter through the piercing has been proposed to cause an inflammatory response that may screen the hole. There is contradictory evidence to support this assertion. The mean age in several of the studies was $>50$ years, when the incidence of post-dural headache puncture is low. Some studies utilised spinal microcatheters, 26G-32G, whereas others used the $18 \mathrm{G}$ Tuohy needle to insert 20G epidural catheters. [17]

Histopathological investigations using long-term intrathecal catheters in animals and people confirmed the existence of an inflammatory response at the catheter site. It would seem unreasonable to compare the impact of a catheter placed on the spot for 24 hours and for many days or weeks. If the insertion intrathecal catheter reduces the post-dural headache rate following inadvertent dural puncture with a Tuohy needle, it might be worth investigating. The use of intrathecal catheters should be excluded from neurological problems, such as cauda equina and infection. In order to treat many various types of headache, the technique used to block the larger occipital nerve (GON) from local anaesthetic has been used and its advantage in the treatment of PDPH has also been observed. Greater occipital nerve blocs (GONB) have been shown to provide symptomatic relief to conservative treatments in patients with PDPH, making it a viable alternative procedure that may be used in patients who reject an epidural blood patch. [19]

GONB is performed by a skilled anaesthesiologist utilising an ultrasound or a landmark method. Local anaesthetics are administered into the region around GON, which are generally rapidly improved clinically by subjective patients [15].

Sphenopalatin ganglion block: sphenopalatin ganglion block (SPGB) is another regional method used rarely for treatment of non-conservative PDPH patients. [19].

On rare circumstances, surgery may be necessary for a dural repair If there is severe headache and nonsurgical therapy is resistant. Evidence of a large CSF 
imagery leak may also indicate a surgical procedure. [16]

\section{Conclusion}

Headache treatment after accidental hard puncture varies. This diversity extends to the literature with various PDPHA definitions and epidural blood patch indications. More worrisome was the absence of follow-up of patients following accidental dural puncture from a survey submitted to Society for Obstetrical Anesthesia and Perinatology. Due to contradictory facts and views, a documented procedure is essential and all departmental members have to be followed. After unintentional dural puncture, the essential components of a successful procedure are as follows: See all patients after birth and at the hospital every day. Provide patients with a phone number if they experience headache. Track all patients with postdural headache puncture symptoms. If a patient cannot attend to everyday tasks, give an epidural blood patch. Consider intrathecal catheter if it is difficult to put it. Consider the preventive epidural blood patch for epidural catheter residing after accidental dural puncture. Do not use a volume higher than $20 \mathrm{~mL}$ while making an epidural blood patch.

\section{References}

[1] A.Ghaleb,A.Khorasani, and D.Mangar, "Postdural puncture headache," Int. J. Gen. Med., vol. 5, p. 45, 2012.

[2] A.Meyer-Bender, A.Kern, B. Pollwein, A. Crispin, and P. M. Lang, "Incidence and predictors of immediate complications following perioperative non-obstetric epidural punctures," BMC Anesthesiol., vol. 12, pp. 1-7, 2012.

[3] L.E.Carrie and P.D.Collins, "29-gauge spinal needles.," BJA Br. J. Anaesth., vol. 66, pp. 145-146, 1991.

[4] A.Bier, "Versuche über cocainisirung des rückenmarkes," Dtsch. Zeitschrift für Chir., vol. 51, pp. 361-369, 1899.

[5] M.Chuanjie Wu . "A multicenter clinical study on treating post-dural puncture headache with an intravenous injection of aminophylline," Pain Physician, vol. 19, pp. E761-E5, 2016.

[6] R.W.Evans, "Complications of lumbar puncture,” Neurol. Clin., vol. 16, pp. 83-105, 1998.

[7] H.C.C.of the I.H.S.(IHS), "The international classification of headache disorders, (beta version)," Cephalalgia, vol. 33, pp. 629-808, 2013.

[8] P.E.Hansen and J.H.Hansen, "DDAVP, a synthetic analogue of vasopressin, in prevention of headache after lumbar puncture and lumbar pneumoencephalography," Acta Neurol. Scand., vol. 60, pp. 183-188, 1979.
[9] D.T.Nguyen and R.R.Walters, "Standardizing management of post-dural puncture headache in obstetric patients: a literature review," Open J. Anesthesiol., vol. 4, pp. 244, 2014.

[10] A.Sabharwal and G.M.Stocks, "Postpartum headache: diagnosis and management," Contin. Educ. Anaesthesia, Crit. Care Pain, vol. 11, pp. 181-185, 2011.

[11] M. R. Alam, M. R. Raheen, K. M. Iqbal, and M. R. A. Chowdhury, "Headache following spinal anaesthesia: a review on recent update," J. Bangladesh Coll. Physicians Surg., vol. 29, pp. 32-40, 2011.

[12] X.B.Ona, D.Osorio, and X.B.Cosp, "Drug therapy for treating post-dural puncture headache," Cochrane database Syst. Rev., vol. 1, pp, 53-61, 2015.

[13] M.Daoud, M.Asfour, and S.Mubashirulhassan, "Missed neostigmineatropine side effects: uncommonly noticed postanesthesia but commonly noticed in other situations," Anesth. Analg., vol. 128, p. e128, 2019.

[14] B.S.Alper and P.R.Lewis, "Treatment of postherpetic neuralgia: a systematic review of the literature," Database Abstr. Rev. Eff. Qual. Rev. [Internet], vol. 1, pp. 31-42, 2002.

[15] M.M.Charsley and S.E.Abram, "The injection of intrathecal normal saline reduces the severity of postdural puncture headache," Reg. Anesth. Pain Med., vol. 26, pp. 301305, 2001.

[16] C.A.Riley and J.E.Spiegel, "Complications following large-volume epidural blood patches for postdural puncture headache. Lumbar subdural hematoma and arachnoiditis: initial cause or final effect?," J. Clin. Anesth., vol. 21, pp. 355-359, 2009.

[17] M.J.Paech, D.A.Doherty, T.Christmas, and C.A.Wong, "The volume of blood for epidural blood patch in obstetrics: a randomized, blinded clinical trial," Anesth. Analg., vol. 113, pp. 126-133, 2011.

[18] S.Devroe, M.Van de Velde, P.Demaerel, and K.Van Calsteren, "Spinal subdural haematoma after an epidural blood patch," Int. J. Obstet. Anesth., vol. 24, pp. 288-289, 2015.

[19] D.Katz and Y.Beilin, "Review of the alternatives to epidural blood patch for treatment of postdural puncture headache in the parturient," Anesth. Analg., vol. 124, pp. 1219-1228, 2017. 